\title{
The sorption characteristics of PAHs onto soils in the presence of synthetic and bio surfactant
}

\author{
J.-H. Chang ${ }^{1}$, Z. Qiang ${ }^{2}$ \& C.-P. Huang ${ }^{3}$ \\ ${ }^{I}$ Department of Environmental Engineering and Management, \\ Chaoyang University of Technology, Taiwan \\ ${ }^{2}$ Environmental Research Center, \\ Department of Civil, Architectural and Environmental Engineering, \\ University of Missouri-Rolla, USA \\ ${ }^{3}$ Department of Civil and Environmental Engineering, \\ University of Delaware, USA
}

\begin{abstract}
Contaminants such as polycyclic aromatic hydrocarbons (PAHs) are of special concern due to their high toxicities and hydrophobicities. The high hydrophobic natures of PAHs enable their strong sorption onto soil or sediments, which makes it difficult for their removal from the environment. This study was to explore the sorption characteristics of PAHs with synthetic nonionic surfactant and biosurfactant in the soil. Two surfactants (one synthetic and one naturally occurring) Triton X-100 and rhamnolipid were used. Results show that the partition coefficient of PAHs in the Triton X-100 system decreases with increasing surfactant concentrations. Throughout the whole concentration range of studied biosurfactant $(0 \sim 30 \mathrm{CMC})$, the partition coefficient of PAHs in rhamnolipid system is roughly constant, around $400.0 \mathrm{~L} / \mathrm{Kg}$. Keywords: sorption, soils, PAHs, surfactant, biosurfactant.
\end{abstract}

\section{Introduction}

The polycyclic aromatic hydrocarbons (PAHs) are of special concern for the natural environment due to their high toxicities and hydrophobicities. On the basis of the characteristic of high hydrophobicity, PAHs are strongly sorbed onto soil or sediments [1]. As such, it is difficult to remove PAHs from the soils. Among many remediation techniques, surfactants have been induced in soil- 
washing, soil-flushing, and pump-and-treat technologies to increase the solubilization of sorbed hydrophobic contaminants [2]. The sorption-desorption process has been considered as the most critical mechanism with respect to the soil remediation. To study the sorption reaction of PAHs in the soil-water system in the presence of surfactants one is able to gain insight into the surfactant remediation process of contaminated soils. The purpose of this investigation is to explore partition coefficients of different PAHs in the presence of surfactants. In addition to the synthetic surfactant, the biosurfactant is also evaluated in this study.

\subsection{Surfactant fundamentals}

Surfactants can be categorized into four groups including cationic, anionic, nonionic and zwitterionic (both cationic and anionic groups). These classifications are typically based on the nature of their head group (hydrophilic head). In aqueous solution, surfactant molecules have the formation of aggregates known as micelles when the surfactant concentrations exceed a certain value. The certain concentration is called the critical micelle concentration, namely, CMC. The CMC value of surfactant is a function of surfactant type (nonionic type generally has lower CMC than ionic one), and system conditions (e.g., temperature, hardness) [3]. Compared to the cosolvent (e.g., alcohols), surfactants exhibit a much higher degree of surface activity and present the high potential for remediation techniques.

In aqueous solution, micelles consist of two portions: one portion is the hydrophilic exterior (the hydrophilic heads are oriented to water phase) and the other is the hydrophobic interior (the hydrophobic tails are oriented to the center of micelles). Since hydrophobic organic compounds can be dissolved into the interior of micelle, the solubilities of organics are then enhanced in the solution. The increased "aqueous solubility" of organic compounds in micelles is referred to as solubilization. The degree of organic solubilization depends on the class and the concentration of surfactants.

\subsection{Biosurfactant}

Biosurfactants are produced by many different bacterial genera. There are a variety of biosurfactants manufactured and most of them can be classified as anionic or nonionic biosurfactants. In addition, biosurfactants can also be classified into several broad groups: glycolipids, lipopeptides, lipopolysaccharides, phospholipids, and fatty acids/neutral lipids [4]. The largest and best-studied group of the biosurfactant is glycolipids, which includes the sophorose-, rhamnose-, trehalose-, sucrose-, and fructose-lipids. In this study, the rhamnolipid biosurfactant has been chosen as the dissolution agent. The production and purification of biosurfactants are difficult, which are controlled by many factors including growth conditions, culturing medium nutrients, temperature, $\mathrm{pH}$, and agitation method [5]. Molecular weight of biosurfactant ranges from approximately 500 to $1,500 \mathrm{mw}$ and $\mathrm{CMC}$ value of biosurfactant typically ranges from 1 to $200 \mathrm{mg} / \mathrm{L}$ [6]. 


\subsection{PAHs sorption-desorpiton phenomena}

PAHs sorption onto the soil in the presence of surfactants can be distributed in three phases including water, micelles, and the soil. During the sorptiondesorption process, there are mainly three reactions occurring such as: (1) the dissolution of PAHs in the micelle phase, (2) the sorption of surfactant in the soil phase, (3) the partition of PAHs between aqueous and soil phase [7]. As all of three reactions reach equilibrium, the partition coefficient is defined as the ratio between the concentration of the PAHs in the soil phase and in the liquid phase (water+micelle), respectively. Although the liquid phase also comprises the other sub-phases including the dissolved organic matter (DOM) and colloids, the effect of DOM and colloids in the liquid phase is relatively small and can be neglected compared to the micelle concentration. The measured partition coefficient is still an apparent rather than true coefficient between aqueous and soil phase.

\section{Methodology}

The soil samples collected from a specific waste site were air-dried and sieved through a No. 10 standard sieve ( $2 \mathrm{~mm}$ openings). Table 1 shows the physicalchemical properties of this soil sample with corresponding analytical methods adopted. The PAHs organic chemicals were purchased from Aldrich Co. The purity of fluorine, phenanthrene, fluoranthene, and pyrene is $98.0 \%$ and that of anthracene is $97.0 \%$. Table 2 lists the major features of these PAHs organic chemicals including molecular formula, molecular weight, saturation water solubility, and $\log \mathrm{K}_{\mathrm{ow}}$. The selected surfactants were Triton $\mathrm{x}-100$ and biosurfactant (rhamnolipid).

Table 1: $\quad$ Basic physical-chemical characteristics of the soil.

\begin{tabular}{lcl}
\hline $\begin{array}{l}\text { Physical-chemical } \\
\text { Characteristics }\end{array}$ & Result & Method \\
\hline Sand (\%) & 14.0 & Hydrometer \\
Silt (\%) & 38.0 & Hydrometer \\
Clay (\%) & 48.0 & Hydrometer \\
pH & 7.6 & In $0.01 \mathrm{M} \mathrm{CaCl}{ }_{2}$ \\
Organic Matter (\%) & 1.7 & Heating at $105^{\circ} \mathrm{C}$ for 2 hours, then \\
& & at $360^{\circ} \mathrm{C}$ for 2 hours \\
\hline
\end{tabular}

Batch sorption experiments were conducted with fluorene, phenanthrene, anthracene, fluoranthene, and pyrene at various CMC values ranging from 1 to 5 . The soil samples were contaminated by PAHs in advance, which concentrations in soils ranged from $25 \mu \mathrm{g} / \mathrm{g}$ to $200 \mu \mathrm{g} / \mathrm{g}$. To a series of glass tubes, $2 \mathrm{~g}$ of PAHs contaminated soil samples and $10 \mathrm{ml}$ of various concentration ( $0 \mathrm{CMC}$ to 30 CMC) surfactant solutions were added. Two surfactants were used to conduct the sorption experiments including Triton $\mathrm{x}-100$ and rhamnolipid. The tubes were placed in a shaker and shaken constantly for 72 hours to reach the 
equilibrium condition. The mixtures were then centrifuged at 2,500 rpm $(1,000$ g) for 10 minutes using a Precision Scientific Co. model K-9 centrifuge to separate the coarse particles from the supernatant. Then, a Sorvall superspeed refrigerated centrifuge model RC-5 was used to remove fine particles in the supernatant at the rotation speed of $12,000 \mathrm{~g}$. The concentration of PAHs organic compounds in the centrate was analyzed with a HPLC/FLD (HewlettPackard, model 1100 series), respectively.

Table 2: The physical properties of fluorene, phenanthrene, fluoranthene, pyrene, and anthracene.

\begin{tabular}{||l|l|l|l|l|l||}
\hline $\begin{array}{l}\text { Physical } \\
\text { Property }\end{array}$ & fluorene & phenanthrene & anthracene & fluoranthene & pyrene \\
\hline $\begin{array}{l}\text { Molecular } \\
\text { Formula }\end{array}$ & $\mathrm{C}_{13} \mathrm{H}_{10}$ & $\mathrm{C}_{14} \mathrm{H}_{10}$ & $\mathrm{C}_{14} \mathrm{H}_{10}$ & $\mathrm{C}_{16} \mathrm{H}_{10}$ & $\mathrm{C}_{16} \mathrm{H}_{10}$ \\
\hline $\begin{array}{l}\text { Molecular } \\
\text { Weight }\end{array}$ & 166.2 & 178.2 & 178.2 & 202.3 & 202.3 \\
\hline $\begin{array}{l}\text { Solubility } \\
(\mathrm{mg} / \mathrm{L})\end{array}$ & 1.84 & 1.09 & 0.06 & 0.23 & 0.13 \\
\hline logK & 4.18 & 4.57 & 4.54 & 5.22 & 5.13 \\
\hline
\end{tabular}

\section{Results and discussion}

Figure 1 shows the sorption isotherms at various Triton $\mathrm{X}-100 \mathrm{CMC}$ values for fluorene, phenanthrene, and anthracene, respectively. Results of fluoranthene and pyrene present the similar trend (not shown here). All of these isotherms can be fitted by linear equations. It implies that the partition process is the main factor for the PAHs distribution in the micelle-soil-water system. The driving forces of partition process are believed as the hydrophobicity of PAHs, surfactant, and soils. Because the partition coefficient is used to describe the competition of PAHs in the liquid and soil phases, the magnitude of various PAHs partition depends not only on their water solubilities but also on the sorption ability of the soils and surfactants. As such, no significant relationship presents between partition coefficient and solubility in all figures.

Figure 1 also shows that partition coefficients of PAHs decreases with increasing the surfactant concentrations. This observation is in agreement with the results reported by Sun and Boyd [8]. It is noticed that the difference between isotherms of blank and $5 \mathrm{CMC}$ is insignificant for these five PAHs compounds. It can be attributed to the sorption of surfactant on soil surface; therefore, the solubilization capacity of PAHs is not significant. In addition, results present that large amount of surfactants were sorbed in soil phase, the sorbed surfactants may increase the sorption capacity of the soil.

Figure 2 shows sorption isotherms at various rhamnolipid $\mathrm{CMC}$ values for fluorene, phenanthrene, and anthracene, respectively. Results of fluoranthene and pyrene with a similar trend are not shown. In the figure, the differences among various surfactant concentrations are insignificant. 


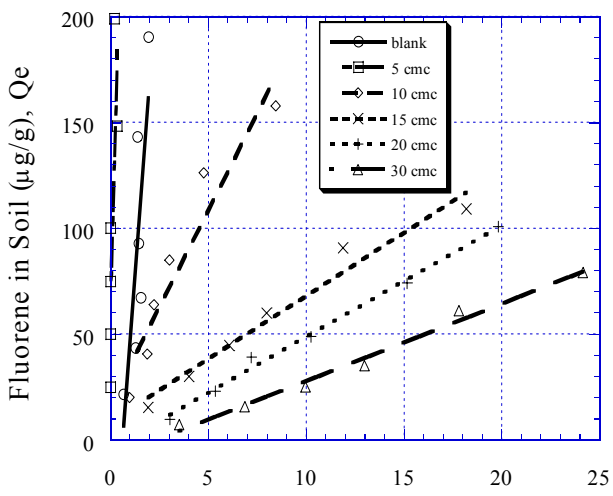

Fluorene in Solution (mg/L), Ce

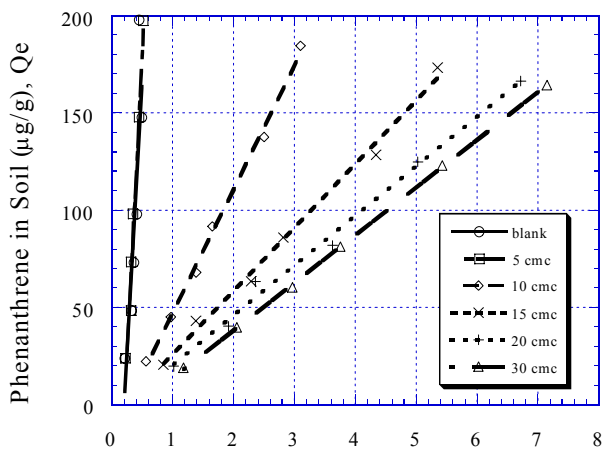

Phenanthrene in Solution $(\mathrm{mg} / \mathrm{L}), \mathrm{Ce}$

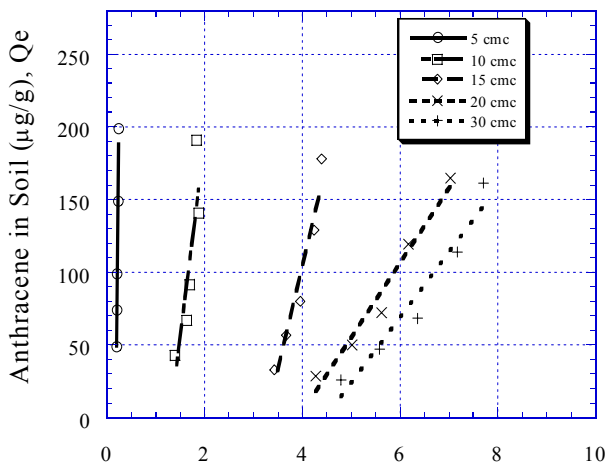

Anthracene in Solution (mg/L), Ce

Figure 1: The PAHs isotherms with various CMC values in Triton $\mathrm{X}-100$ solution-soil system such as fluorine, phenanthrene, anthracene. 
148 Geo-Environment and Landscape Evolution II
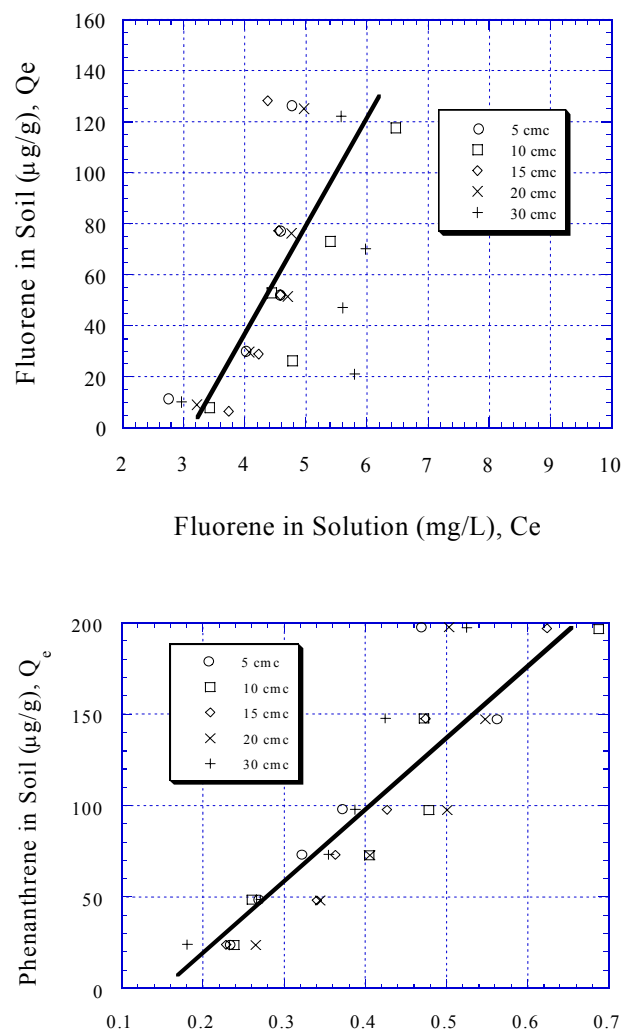

Phenanthrene in Solution $(\mathrm{mg} / \mathrm{L}), \mathrm{Ce}$

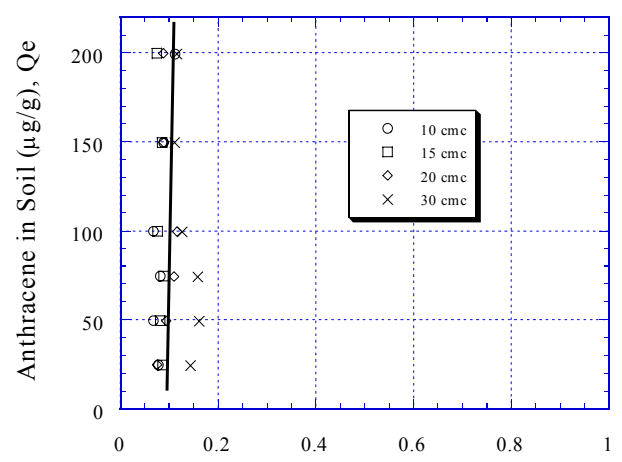

Anthracene in Solution $(\mathrm{mg} / \mathrm{L}), \mathrm{Ce}$

Figure 2: The PAHs isotherms with various CMC values in rhamnolipid solution-soil system such as fluorine, phenanthrene, anthracene. 
Most equilibrium concentrations of PAHs in these data are below $1 \mathrm{mg} / \mathrm{L}$, which are far lower than their solubilities $(10 \sim 20 \mathrm{mg} / \mathrm{L})$ under the same rhamnolipid concentration in water. This implies that most biosurfactant molecules are sorbed in soils and just few in the solution to aid dissolving PAHs compounds from soil to aqueous phase. However, these isotherms still can roughly be fitted by linear equations. Throughout whole concentration range studied of biosurfactant concentration $(0 \sim 30 \mathrm{CMC})$, the partition coefficient of PAHs in rhamnolipid system is constant, around $400.0 \mathrm{~L} / \mathrm{Kg}$.

\section{Conclusion}

The partition coefficient of PAHs between soil and micelle aqueous phase can be described by the linear equations regardless of synthetic or bio surfactants. In the Triton X-100 system, the partition coefficient of PAHs decreases with the surfactant concentrations. In addition, Triton X-100 is sorbed in soil; the sorbed surfactants may influence the sorption capacity of the soil. Throughout whole biosurfactant concentration $(0 \sim 30 \mathrm{CMC})$, the partition coefficient of PAHs in rhamnolipid system can be regarded as a constant, around $400.0 \mathrm{~L} / \mathrm{Kg}$. The biosurfactant also exhibits strong attraction onto the soil.

\section{References}

[1] Karickhoff, S.W., Organic Pollutant Sorption in Aquatic System. Journal of Hydraulic Engineering, 10(6), pp. 707-735, 1984.

[2] Mackay, D.M. \& Cherry, J.A., Groundwater Contamination: Pump-andTreat Remediation. Environmental Science and Technology, 23(6), pp. 630-636, 1989.

[3] Rosen, M.J., (eds). Surfactants and Interfacial Phenomena, John Wiley \& Sons Inc.: New York, 1989.

[4] Fiechter A., Biosurfactants: Moving towards Industrial Application. Trends Biotech, 10, pp. 208-217, 1992.

[5] Miller R.M., Biosurfactant-Facilitated Remediation of MetalContaminated Soils. Environmental Health Perspective, 103(Suppl 1), pp. 59-62, 1995.

[6] Lang, S. \& Wagner, F., (eds). Structure and Properties of Biosurfactants. In: Biosurfactants and Biotechnology, New York, 1994.

[7] Shaoo D., Smith, J.A., Imbrigiotta, T.E., \& Mclellan, H.M., SurfactantEnhanced Remediation of a Trichloroethene -Contaminated Aquifer. 2. Transport of TCE. Environmental Science and Technology, 32(11), pp. 1686-1693, 1998.

[8] Sun, S. \& Boyd, S.A., Sorption of Nonionic Organic Compounds in SoilWater Systems Containing Petroleum Sulfonate-Oil Surfactants. Environmental Science and Technology, 27(9), pp. 1340-1346, 1993. 